\title{
Porous-core honeycomb bandgap THz fiber
}

Nielsen, Kristian; Rasmussen, Henrik K.; Jepsen, Peter Uhd; Bang, Ole

Published in:

Optics Letters

Link to article, DOI:

10.1364/OL.36.000666

Publication date:

2011

Document Version

Publisher's PDF, also known as Version of record

Link back to DTU Orbit

Citation (APA):

Nielsen, K., Rasmussen, H. K., Jepsen, P. U., \& Bang, O. (2011). Porous-core honeycomb bandgap THz fiber. Optics Letters, 36(5), 666-668. https://doi.org/10.1364/OL.36.000666

\section{General rights}

Copyright and moral rights for the publications made accessible in the public portal are retained by the authors and/or other copyright owners and it is a condition of accessing publications that users recognise and abide by the legal requirements associated with these rights.

- Users may download and print one copy of any publication from the public portal for the purpose of private study or research.

- You may not further distribute the material or use it for any profit-making activity or commercial gain

- You may freely distribute the URL identifying the publication in the public portal

If you believe that this document breaches copyright please contact us providing details, and we will remove access to the work immediately and investigate your claim 


\title{
Porous-core honeycomb bandgap THz fiber
}

\author{
Kristian Nielsen, ${ }^{1}$ Henrik K. Rasmussen, ${ }^{2}$ Peter Uhd Jepsen, ${ }^{1}$ and Ole Bang ${ }^{1, *}$ \\ ${ }^{1}$ DTU Fotonik, Department of Photonics Engineering, Technical University of Denmark, DK-2800 Kongens Lyngby, Denmark \\ ${ }^{2}$ DTU Mekanik, Department of Mechanical Engineering, Technical University of Denmark, DK-280o Kongens Lyngby, Denmark \\ ${ }^{*}$ Corresponding author: oban@fotonik.dtu.dk
}

Received December 9, 2010; accepted January 9, 2011;

posted February 1, 2011 (Doc. ID 139341); published February 23, 2011

\begin{abstract}
In this Letter we propose a novel (to our knowledge) porous-core honeycomb bandgap design. The holes of the porous core are the same size as the holes in the surrounding cladding, thereby giving the proposed fiber important manufacturing benefits. The fiber is shown to have a 0.35-THz-wide fundamental bandgap centered at 1.05 THz. The calculated minimum loss of the fiber is $0.25 \mathrm{~dB} / \mathrm{cm}$. (C) 2011 Optical Society of America

OCIS codes: $\quad 060.5295,260.3090$.
\end{abstract}

Terahertz radiation has attracted widespread attention in recent years because of its unique possibilities in spectroscopy and imaging [1]. Terahertz waveguides have consequently also attracted attention, both for distributing the radiation and as functional devices [2]. Apart from hollow metallic and glass waveguides $[3, \overline{4}]$, the material of choice when making terahertz waveguides is polymer, i.e., poly(methyl-methacrylate) (PMMA) [5], Teflon [6], high-density polyethylene (HDPE)[7,8], and Topas [יㅜㄹ. The reasons for this material choice are that polymers have the lowest loss of most materials in the $\mathrm{THz}$ range and they are for the most part easy to machine.

Currently there is much research invested in lowering the loss by forcing a large part of the radiation to propagate in air while still being confined to a waveguide, i.e., subwavelength fiber [8], porous fibers $[10,11]$, and hollow-core fibers. Of these, the hollow-core fiber is the least sensitive to outside perturbations, and thus the fiber can be handled without altering the propagation properties. Hollow-core fibers come in different designs, such as the simple tube waveguide (loss $0.95 \mathrm{~dB} / \mathrm{m}$ ) [3] , the antiresonance waveguide (loss $<0.0434 \mathrm{~dB} / \mathrm{cm}$ ) [12], the Bragg fiber (loss $0.9 \mathrm{~dB} / \mathrm{cm}$ ) [5], and the hollow-core bandgap fiber (loss $1 \mathrm{~dB} / \mathrm{m}$ ) [13,14]. The bandgap fibers are typically designed using a tightly packed triangular structure in which the holes are highly inflated to give a high air fill fraction. The high air fill fraction, in combination with a large air defect in the core, is needed in order to achieve an efficient and broad bandgap [14]. Creating fibers with different sized air holes and also fibers with a very large air fill fraction is a manufacturing challenge. When the holes have different sizes, the interaction between the holes during manufacturing is asymmetric, causing the smaller holes to deform. The imbalance between the holes can to some extent be helped by applying air pressure on the holes during manufacturing [15]. Here we propose dealing with these issues by using the honeycomb structure as our bandgap basis and, instead of having one big hole as core defect, we introduce a porous core where the holes are of the same size as the holes in the cladding. Honeycomb bandgaps are relatively larger than triangular bandgaps, and at comparable structure sizes the honeycomb bandgap is at lower frequencies than the triangular bandgap [16], which can be exploited to make a thinner fiber. The thickness of the fiber is one of the factors limiting the applications of terahertz fibers. In order to be flexible and for compactness the fiber should be as thin as possible. For completeness we compare the performance of the porous-core honeycomb fiber with that of a hollow-core fiber with the same honeycomb cladding structure.

The honeycomb bandgap fiber designs are shown in Fig. 1. In the porous-core fiber all the holes have the same diameter $d$. In the hollow-core fiber the core is created by removing seven honeycomb cells, resulting in a hollow core of the same size as the effective core of the porouscore fiber.

The fiber is considered to be made of Topas with a constant refractive index of 1.526 [9]. Apart from nearly zero material dispersion [9], Topas, as opposed to many other polymers, does not absorb water [17]. The structure is chosen to have a pitch of $\Lambda=250 \overline{\mu m}$ and hole-to-pitch ratio of $d / \Lambda=0.55$, as this results in a fundamental bandgap near $1 \mathrm{THz}$. The bandgap of the cladding unit cell is calculated using the MIT Photonic-Bands package (MPB) [18]. The resulting bandgaps are shown in gray in Fig. 2 . In order to take the finite cladding structure into consideration, the calculations on the fiber are made using the commercially available finite-element mode solver COMSOL. The fundamental core mode of a four-ring fiber for both bandgaps is also shown in Fig. 2. Since the modeled fibers have a finite cladding size, the resulting bandgap is different from the one found using MPB. Therefore some of the core modes extend slightly outside the bandgap indicated in gray in Fig. 2. The intensities of the three mode insets in Figs. 2(a)-2(c) cannot be compared because they are normalized individually. In order to define the core mode, the fraction of power in the core is calculated; the core is defined as a circle of radius $300 \mu \mathrm{m}$.

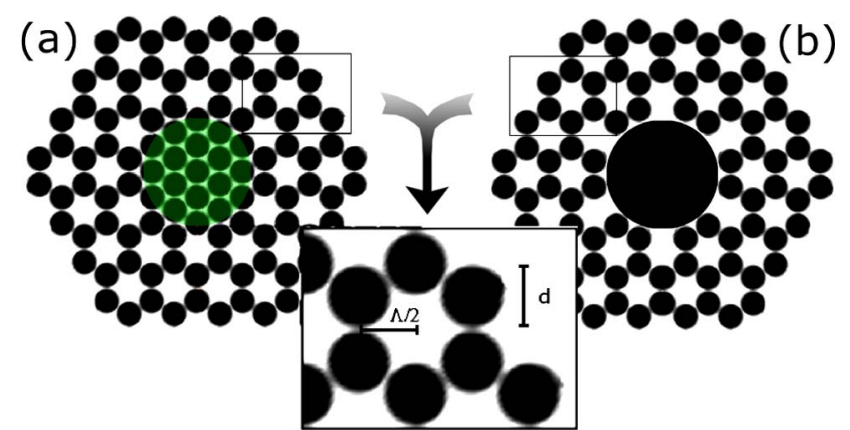

Fig. 1. (Color online) Honeycomb fiber with (a) a seven-hole porous core and (b) a seven-cell hollow core. Dark regions represent air. 
The dashed curves in Fig. 2 have a fraction of power in the core larger than 55\%, while the solid curve has an overlap larger than $25 \%$. The second bandgap has several higher-order core modes. These are not included because they all lie close to the bandgap edge, making them sensitive to perturbations and therefore unfit as guiding modes.

The bandgaps only dip below the airline in a narrow frequency range due to the low air filling fraction. Even so, the hollow-core fiber supports a guided mode. However, this mode has most of the field guided in the six rods closest to the core, as seen in Fig. 2(c); this result corresponds to earlier findings for silica fibers [19]. This leads to a low fraction of power in the core. Consequently the mode cutoff for the hollow-core fiber is defined as when the fraction of power in the core is less than $25 \%$. Even though the second bandgap also dips below the air line, the hollow-core fiber does not support any core mode in the second bandgap. The slope of the mode lines in Fig. 2 indicate that the porous-core fiber is less dispersive than the hollow-core fiber, and they also show that the porous-core fiber has a wider transmission window than the hollow-core fiber.

The behavior of the fundamental core mode is dependent on the extent of the cladding. In order to investigate this relation in more detail, we consider cladding sizes of two, three and four rings for the porous-core fiber. The fraction of power in the core in the first bandgap of the three cases is shown in Fig. 3(a). The four-ring fiber (dashed curve) gives the most clean bandgap, whereas the two-ring (dash-dotted curve) and three-ring (dotted curve) fibers have large confinement dips in the bandgap. The hollow-core fiber (solid black curve) has a four-ring cladding and confines the field poorly to the core. A fourring fiber with the given pitch would result in a $3 \mathrm{~mm}$ thin fiber, half the thickness of the previously reported Bragg fiber [5].

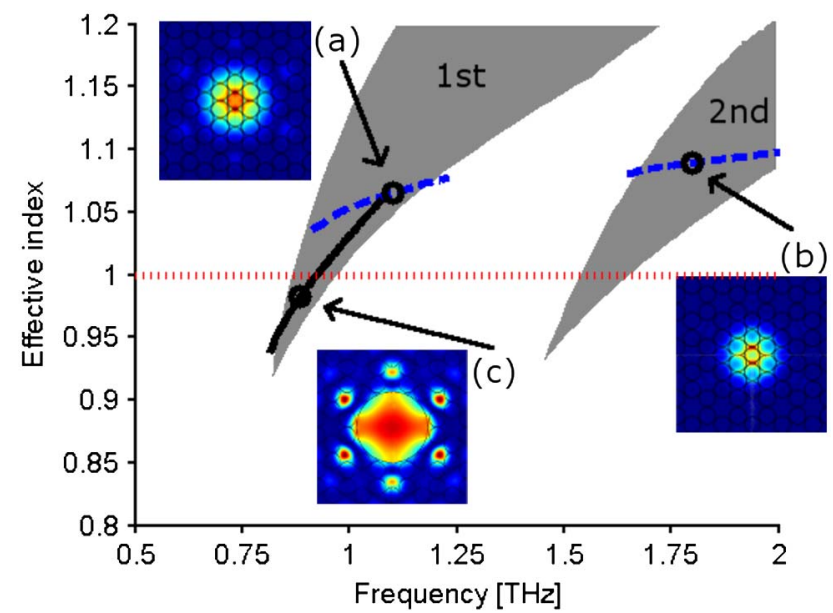

Fig. 2. (Color online) The calculated bandgap is shown in gray, the effective index of the core mode of the porous fiber are shown as blue dashed curves, and the effective index of the core mode of the hollow-core fiber is shown as a solid black curve. The dotted straight line indicates the air line. The insets show the $E_{y}$ field of the modes of the porous fiber at (a) $1.1 \mathrm{THz}$ and (b) $1.8 \mathrm{THz}$ and (c) the $E_{y}$ field of the mode of the hollowcore fiber at $0.885 \mathrm{THz}$.
The effective material loss of the fiber $\alpha_{\text {eff }}$ can be estimated using [므]

$$
\alpha_{\mathrm{eff}}=\frac{\left(\epsilon_{0} / \mu_{0}\right)^{1 / 2} \int_{A_{\text {mat }}} n \alpha_{\text {mat }}|\mathbf{E}|^{2} \mathrm{~d} A}{\operatorname{Re}\left\{\int_{A_{\text {all }}} \mathbf{E} \times \mathbf{H}^{*} \cdot \mathbf{z d} A\right\}}=\alpha_{\mathrm{mat}} \eta,
$$

where Re denotes the real part, $n$ is the refractive index of the material, and $\alpha_{\text {mat }}$ is the material loss. $\mathbf{E}$ is the electric field component and $\mathbf{H}^{*}$ is the complex conjugated magnetic field component. $\mathbf{z}$ is the unit vector in the $z$ direction. The integration in the numerator is only performed over the solid material $\left(A_{\text {mat }}\right)$ because the propagation loss of terahertz radiation in air is negligible. As the material loss is spatially uniformed, the effective loss $\left(\alpha_{\text {eff }}\right)$ is given by the product of the material loss and the fraction of power in the material $(\eta)$. The low loss approximation is applied so that the field components used in the integrals are calculated for a structure with no loss. The resulting $\eta$ is shown in Fig. 3(b), which shows that in the middle of the porous-core fiber bandgap the effective loss is approaching $20 \%$ of the bulk loss. The bulk loss at $1 \mathrm{THz}$ is $1 \mathrm{~dB} / \mathrm{cm}$ [9], giving an effective loss of $\alpha_{\text {eff }}=0.20 \mathrm{~dB} / \mathrm{cm}$.
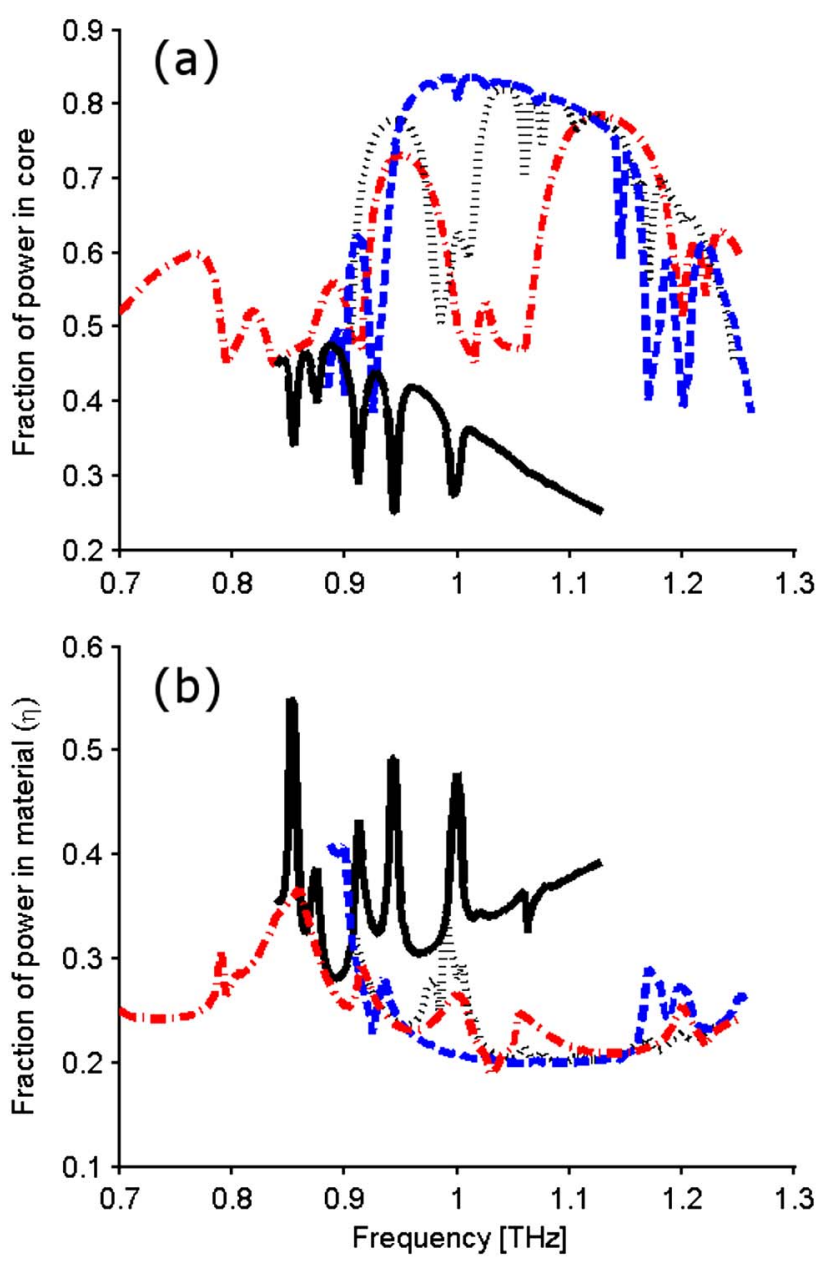

Fig. 3. (Color online) (a) Fraction of power localized in the core of the fiber for two-ring (red dash-dotted curve), three-ring (black dotted curve), and four-ring (blue dashed curve) porouscore fibers and the hollow-core (black solid curve) fiber. (b) Calculated fraction of power in the material $(\eta)$ for all fibers. 


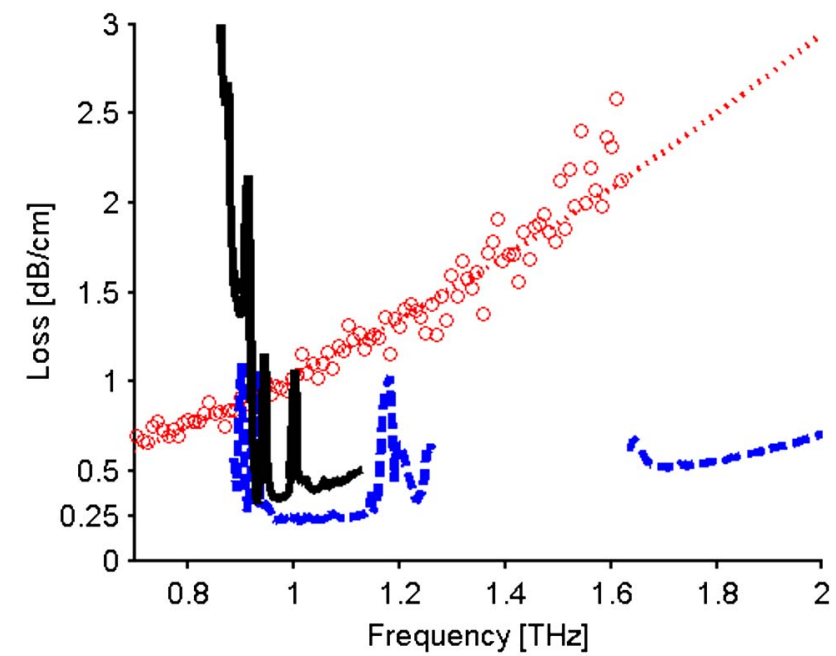

Fig. 4. (Color online) Total loss of the porous-core (blue dashed curve) and hollow-core (black solid curve) four-ring fibers. Bulk loss is shown as red circles and the polynomial fit to the bulk loss is shown as a red dotted curve.

A second loss mechanism in fibers is the confinement loss. This loss is normally much smaller than the material loss, but at the bandgap edges it becomes significant. Using COMSOL it is possible to model a realistic fiber, and by applying a perfectly matched layer around the fiber with a refractive index of 1 (air), the confinement loss can be calculated. In Fig. 4 the confinement loss is added to the effective material loss to produce the total loss for the four-ring fibers. The material loss is the measured bulk loss of Topas [9], shown as red circles. The polynomial fit used in the calculation is shown as a red dotted line.

At the edges of the bandgap the confinement loss increases for both fibers, and the confinement loss is also large in the ranges where the fraction of power in the core is low. Even though the hollow-core fiber supports a mode confined to the low loss air core, a large part of the power is still propagating in the material, giving a high effective loss. Additionally the hollow-core fiber is seen to suffer from excessive confinement loss. The porous-core fiber also supports a mode in the second bandgap with losses at around $0.5 \mathrm{~dB} / \mathrm{cm}$.

In conclusion, we have designed and investigated a porous-core and a hollow-core honeycomb bandgap fiber. The advantage of the porous-core fiber is that it is easier to manufacture than the hollow-core fiber, because all the holes have the same size. Through numerical calculations we showed that the porous-core fiber has a wider bandgap than the corresponding hollow-core fiber and lower total propagation loss. The calculations showed that four rings of cladding are required to get a useful bandgap, and in this bandgap the confinement loss is small compared to the material loss. A four-ring fiber with the given pitch of $250 \mu \mathrm{m}$ could be as thin as $3 \mathrm{~mm}$ in diameter. This fiber is realistically manufacturable and would be half the thickness of the only previous experimentally reported bandgap fiber [5] and, with a loss of $0.25 \mathrm{~dB} / \mathrm{cm}$, have four times lower loss.

\section{References}

1. P. U. Jepsen, D. G. Cooke, and M. Koch, Laser \& Photon. Rev. 5, 124 (2011).

2. K. Nielsen, H. K. Rasmussen, P. U. Jepsen, and O. Bang, Opt. Lett. 35, 2879 (2010).

3. B. Bowden, J. A. Harrington, and O. Mitrofanov, J. Appl. Phys. 104, 093110 (2008).

4. B. Bowden, J. A. Harrington, and O. Mitrofanov, Opt. Lett. 32, 2945 (2007).

5. C. S. Ponseca, Jr., R. Pobre, E. Estacio, N. Sarukura, A. Argyros, M. C. J. Large, and M. A. van Eijkelenborg, Opt. Lett. 33, 902 (2008).

6. M. Goto, A. Quema, H. Takahashi, S. Ono, and N. Sarukura, Jpn. J. Appl. Phys. Part 2 43, L317 (2004).

7. H. Han, H. Park, M. Cho, and J. Kim, Appl. Phys. Lett. 80, 2634 (2002).

8. L. J. Chen, H. W. Chen, T. F. Kao, J. Y. Lu, and C. K. Sun, Opt. Lett. 31, 308 (2006).

9. K. Nielsen, A. J. L. Adam, P. C. M. Planken, H. K. Rasmussen, O. Bang, and P. U. Jepsen, Opt. Express 17, 8592 (2009).

10. A. Hassani, A. Dupuis, and M. Skorobogatiy, Opt. Express 16, 6340 (2008).

11. S. Atakaramians, A. V. Shahraam, B. M. Fischer, D. Abbott, and T. M. Monro, Opt. Express 16, 8845 (2008).

12. J. Y. Lu, C. P. Yu, H. C. Chang, H. W. Chen, Y. T. Li, C. L. Pan, and C. K. Sun, Appl. Phys. Lett. 92, 064105 (2008).

13. R. F. Cregan, B. J. Mangan, J. C. Knight, T. A. Birks, P. St. J. Russell, P. J. Roberts, and D. C. Allan, Science 285, 1537 (1999).

14. L. Vincetti, Microw. Opt. Technol. Lett. 51, 1711 (2009).

15. A. Argyros, M. A. van Eijkelenborg, M. C. J. Large, and I. M. Bassett, Opt. Lett. 31, 172 (2006).

16. J. Broeng, S. E. Barkou, A. Bjarklev, J. C. Knight, T. A. Birks, and P. S. Russell, Opt. Commun. 156, 240 (1998).

17. G. Emiliyanov, J. B. Jensen, O. Bang, P. E. Hoiby, L. H. Pedersen, E. M. Kjær, and L. Lindvold, Opt. Lett. 32, 460 (2007).

18. S. G. Johnson and J. D. Joannopoulos, Opt. Express 8, 173 (2001).

19. M. Yan and P. Shum, IEEE Photon. Technol. Lett. 17, 64 (2005).

20. A. W. Snyder and J. Love, Optical Waveguide Theory (Kluwer Academic, 2000). 